\title{
APORTACIONES AL CONOCIMIENTO FLORISTICO DE LA SIERRA DE ARACENA (HUELVA, ESPAÑA)
}

\author{
J. RIVERA \& B. CABEZUDO
}

\begin{abstract}
RESUMEN: Se ha realizado un catálogo florístico de la Sierra de Aracena (Huelva, España), en el que se citan 900 táxones de plantas vasculares.

SUMmARY: A check-list of the Sierra de Aracena (Huelva, Spain) is presented. A total of 900 vascular plants have been identificated.
\end{abstract}

\section{INTRODUCCION}

La Sierra de Aracena, extremo suroccidental de Sierra Morena, se encuentra situada en el norte de la provincia de Huelva (España) y está formada por un cinturón montañoso que se extiende desde el límite con la provincia de Sevilla hasta el río Chanza, frontera natural con Portugal. Es una zona poco estudiada corológicamente, pues son muy escasos los trabajos que tratan sobre ella (Rivas Goday, 1964; Rivera \& Cabezudo, 1981; Cabezudo 1978a y 1978b; Salvo \& Cabezudo, 1984; Rivas-Martínez, 1979, etc.). Hemos intentado, con esta aportación, completar una laguna importante en el conocimiento florístico de Andalucía y del SW ibérico.

Altitudinalmente está comprendida entre los $400 \mathrm{~m}$ de la zona basal y los 960 en su parte central. Destacan entre otros Pico Castaño $(960 \mathrm{~m})$, Pico de Almonaster $(915 \mathrm{~m})$, Pico de San Ginés $(867 \mathrm{~m})$, Cabezo del Cojo $(800 \mathrm{~m})$ y Pico de Aroche $(721 \mathrm{~m})$.

La situación geográfica y la orografía determinan la existencia en la Sierra de dos pisos bioclimáticos, uno termomediterráneo, entre los 400-600 m, y otro mesomediterráneo por encima de los $600 \mathrm{~m}$ (Rivas-Martínez, 1983), los cuales, combinados con los ombroclimas subhúmedo y húmedo, influyen en la variabilidad de su flora y vegetación. Las principales estaciones meteorológicas estudiadas han sido: Aracena $\left(\mathrm{P}=1104 \mathrm{~mm} ; \mathrm{T}=14,8^{\circ} \mathrm{C}\right)$, Aroche $(\mathrm{P}=840 ; \mathrm{T}=16,2)$, Zufre $(\mathrm{P}=$ 831; $\mathrm{T}=16)$, Calañas $(\mathrm{P}=1020 ; \mathrm{T}=18)$, Almonaster la Real ( $\mathrm{P}=932 ; \mathrm{T}=$ 16,6), Cabeza Rubias ( $P=932 ; \quad T=15,6)$, Valverde del Camino ( $P=792$; $\mathrm{T}=19,4)$, Cala $(\mathrm{P}=670 ; \mathrm{T}=17)$, Jabugo $(\mathrm{P}=1150)$ y Cortegana (1160). Geológicamente la mayor parte de la sierra se caracteriza por la presencia de una serie metamórfica de pizarra y esquistos, exis- 
tiendo un núcleo central de calizas marmóreas duras y algunos enclaves graníticos. Lo accidentado del terreno implica la formación general de suelos pocos desarrollados, muy erosionables y ácidos o ligeramente alcalinos. En la zona de relieve llano-ondulado, se originan suelos más profundos que permiten un mejor desarrollo de la vegetación arbórea.

Corológicamente la zona estudiada está incluida en la Región Mediterránea, formando parte del sector Mariánico-Monchiquense, de la provincia Luso-Extremadurense (Rivas-Martínez, 1982a). Para la confección del catálogo hemos realizado excursiones periódicas durante dos años y que han dado como resultado la recolección e identificación de cerca de un millar de táxones que suponen una aportación considerable al conocimiento florístico del SW de la Península Ibérica.

Para cada especie indicamos la frecuencia de aparición en la zona ( $\mathrm{A}=$ =Abundate; $\mathrm{F}=$ Frecuente; $\mathrm{O}=$ Ocasional; $\mathrm{R}=$ Rara y $\mathrm{Vr}=$ Muy rara), comportamiento bioclimático habitual $(\mathrm{T}=$ Termomediterráneo; $\mathrm{M}=\mathrm{Me}-$ somediterráneo) y mediante cuadrícula UTM $(10 \times 10)$ las zonas donde cada taxon ha sido recolectado.

El catálogo ha sido ordenado según criterios de Flora Europaea (Tutin \& cols., 1964-80). Todo el material mencionado se encuentra depositado en los Herbarios de las Universidades de Sevilla (SEV) y Málaga (MGC).

Aunque la vegetación natụral se encuentra muy deteriorada por la presión agrícola y ganadera, existen zonas muy concretas que nos ofrecen una muestra de las comunidades climax arbóreas que existieron en la Sierra. Son varias las series de vegetación que se pueden reconocer:

1. Serie mesomediterránea Luso-Extremadurense de Quercus pyrenaica Willd. - Arbuto-Quercetum pyrenaicae sigmetum (Rivas-Martínez, $1982 \mathrm{~b})$-. Se asienta en las zonas más altas y húmedas, estando sustituida en la mayor parte de su areal potencial por castañares.

2. Serie mesomediterránea Luso-Extremadurense subhúmeda de Quercus suber L. - Sanquisorbo-Quercetum suberis sigmetum -. Ocupa las zonas de suelos profundos y húmedos de la parte media de la Sierra. En la actualidad se encuentra representado en la mayor parte de su areal por madroñales y brezales.

3. Serie mesomediterránea Luso-Extremadurense silicícola de Quercus rotundifoliae Lam. - Pyro-Quercetum rotundifoliae sigmetum -. Ocupa las zonas basales ácidas y subhúmedas de la Sierra. En la actualidad se encuentra representado en su mayor parte por jarales.

4. Serie termomediterránea mariánica seca silicicola de Quercus rotundifoliae Lam. - Myrto-Quercetum rotundifoliae sigmetum (RivasMartínez, 1982b) - . Muy puntual en la zona, ocupa algunos enclaves térmicos de la parte inferior y se encuentra representada en la actua lidad por jarales termófilos. 


\section{CATALOGO FLORISTICO}

PTERIDOPHYTA

\section{SELAGINELLACEAE}

Selaginella denticulata (L.) Link, $F, T(M)$, $Q B$ 09, QB 99, PB 99, QC 00, QB 39, QB 29.

\section{ISOETACEAE}

Isoetes velata $A . B r ., V r, T, P B 66$.

Isoetes durieui Bory, $\mathrm{Vr}, \mathrm{M}, \mathrm{PB} 89$.

Isoetes histrix Bory, F, T, QB 19, QB 08, PB 99, QB 39, QB 28.

\section{EQUISETACEAE}

Equisetum telmateia Ehrh., 0, T(M), PB 99.

Equisetum ramosissimum Desf., F, T, QC 00, PC 80 .

OPHIOGLOSSACEAE

Ophioglossum lusitanicum L., R, I, QB 19 y 28 . OSMUNDACEAE

Osmunda regalis L., R, M, QB 09, PB 89.

\section{SINOPTERIDACEAE}

Cheilanthes guanchica Bolle, Vr, M, QB 19 y 09.

Cheilanthes pteridioides (Reichard) C. Chr., $R, T(M), Q C$ 00, QB 09, QB 19.

Cheilanthes maderensis Lowe, 0, T(M), QB 09, QB 39, PB 39.

Cheilanthes hispanica Mett., R, T, QB 39, QC 00 .

Cheilanthes tinaei Tod., 0, T, QB 29, PB 99, QB 19, QB 39 .

Cosentinia vellea (Ait) Tod., 0, $T(M), Q B 19$, QB 39, QB 09.

\section{ADIANTACEAE}

Adiantum capillus-veneris L. $F, M(T), Q B 19$, QB 09, QB 29, PC 70, QC 39.

\section{GYMNOGRAMMACEAE}

Anogramma leptophylla (L.) Link, 0, T(M), QB 09, PC 70, PC 90, QB 29 QB 18.

\section{HYPOLEPIDACEAE}

Pteridium aquilinum ( $L$.) Kuhn, $A, M(T), Q B$ 09, QB 19, QC 10, PB 89, QB 29, PB 99.

\section{ASPLENIACEAE}

Ceterach offioinarum $D C ., F, T(M), Q B$ 09, PB 99, QB. 19, QB 28.

Asplenium trichomanes $L$., subsp. quadrivalens Meyer, A, M(T), QB 09, QC 00, PB 99.
Asplenium petrarchae(Guerin) $D C_{\text {_ }}, V_{r}, M, Q B$ 19.

Asplenium onopteris L., F, M, QB 09, PB 99, PB 09, PB 89.

Asplenium billotii $F$. Schultz, $0, M(T), Q C 00$, QB 09, PB 99, PB 89.

\section{ATHYRIACEAE}

Athyrium felix-femina (L.) Roth., R, M, QC 00, PB 89, PC 80 .

Cystopteris fragilis (L.) Bernh., R, M, QB 19, QC 00.

Cystopteris viridula (Desv.) Desv., Vr, M, QC 00 .

\section{ASPIDIACEAE}

Dryopteris affinis (Lowe) Fraser-Jenkins ssp. affinis, R, M, PB 89, QB 09 .

Polystichium setiferum (Forskal) Woynar, $\mathrm{Vr}$, M, QB 09, PB 99.

BLECHNACEAE

Blechnum spicant (L.) Roth., R, M. QC 00, PB 89, PB 88.

\section{POLYPODIACEAE}

Polypodium cambricum L., 0, M, QB 19, PB 99, QB 09, QC 00.

\section{SPERMATOPHYTA \\ DICOTILEDONEAS}

SALICACEAE

Salix fragilis L., F, T(M), QB 09, PB 89, PC 90 .

Salix pedunculata Desf., F, T(M), PC 70, QB 39, QB 29, QB 28, QB 18.

Salix atrocinerea Brot., $F, M(T), P C$ 90, QB 09, QB 28, QB 29, PB 99.

Populus alba L., $0, M(T), P B 99$,

Populus nigra L., F, $T(M), Q C 00, Q B 39, P C 80$, PB 99, PC 70, QB 29.

\section{BETULACEAE}

Alnus glutinosa (L.) Gaertner, F, M(T), PB 99, QB 29, PC 70, QC 00, PB 88, PB 89.

\section{CORYLACEAE}

Corylus avellana L.; R, A, QB 19.

\section{FAGACEAE}

Castanea sativa Miller, F, M, QB 09, PB 99, QC 00, QB 09.

Quercus coccifera L., A, T(M), QB 09, PB 99, QB 19, QB 38, QB 29. 
Quercus rotundifolia Lam. A, T(M), QB 09, PC 90. PB 99, QB 08, PB 89, QB 39.

Quercus suber L., F, M(T), QB 18, PB 89, PB 99.

Quercus pirenaica Willd., 0, M, QB 09, QB 19, QC 00 .

Quercus canariensis Willd., 0, M, QC 00, PB 99, QB 29, QB 09, PC 80, PB 89, QC 10, QB 19.

Quercus faginea Lam., $0, M, Q B 29, Q B$ 09, $P B$ 89, PB 99, OC 00, PC 80.

Quercus fruticosa Brot., R, M, QB 09.

\section{MORACEAE}

Ficus carica L., 0 , I(M), PB 99, PB 89, QB 19 , PC 80.

UR TICACEAE

Urtica dioica L., $0, M(\mathrm{~T}), Q B$ 29, PB $89, Q C$ 00 .

Urtica urens L., F, I(M), QB 29, QC 00, PB 99.

Urtica dubia Forskal, F, T, QB 39.

Parietaria diffusa Mert. \& Koch, 0, M(I), QB 39, QB 09, QB 19.

Parietaria mauritanica Durieu, R, M, QC 00.

\section{SANTALACEAE}

0syris alba L., F, M(T), QB 09, QB 19, QB 39, PC 80.

\section{ARISTOLOCHIACEAE}

Aristolochia baetica L., R, I, QB 17.

Aristolochia longa L., F, M, QB 09, QB 19, PB 99, PB 89, QC 00.

\section{RAFLES IACEAE}

Cytinus hypocistis (L.) L., $\mathbf{0}, \mathrm{T}(\mathrm{M}), \mathrm{QB}$ 09, PB 89, QC 00, PB 99, QB 08.

\section{POLYGONACEAE}

Polygonum persicaria L., $0, T(M), Q B 29, Q B$ 19, PB 89, PC 80, PB 99, PC 70.

Polygonum aviculare L., A, I(M), $Q B$ 19, $P B$ 89, $P B$ 99, QB 29, QB 09, PC 99.

Bilderdykia convolvulus (L.) Dumort, VR, M, QC 00 .

Bilderdykia aubertii (L.Henry) Moldenke, 0 , M, QB 09, QB 19.

Rumex acetosella L., F, T(M), QB 29, QC 00 , PC 81, QB 09, QB 19, QC 10.

Rumex induratus Boiss. E Reuter, $F, T(M), Q B$ 29, QB 19, PB 99, PC 00, QB 09, PC 70 .

Rumex conglomeratus Murray, $F, T(M), Q C 10$, QB 29, QB 09, QC 00, PC 90, QB 19, PB 99, PB 89.

Rumex pulcher L., $A, T(M), P B$ 99, PC 81, QB 19, QC 10, QB 29.

Rumex bucephalophorus L., $A, T(M), Q B$ 19, PC 81, QB 09, QC 00, QB 29, PC 90, QC.10.
CHENOPODIACEAE

Chenopodium opulifolium Schrader, $0, T(M), P B$ 99, $P B$ 89, $Q B$ 29, QB 39, QB 19, QB 09, $P C 90$.

Atriplex rosea L., R, M, PC 90.

Atriplex patula L., R, M, PC 90 .

\section{AMARANTHACEAE}

Amaranthus hibridus L., R, M, QB 19.

Amaranthus albus L., F, T(M), QB 38, PC 90, PC 80, PB 99.

Amaranthus cruentus L., $F, T(M), Q B 29, Q B 00$, QB 09, PC 90, PC 70, PB 89, QB 19, PB 88.

Amaranthus retroflexus L., R, M, QB 19.

\section{PHYTOLACACEEAE}

Phytolaca americana L., R, M, PB 88, QB 09, QC 00 .

\section{PORTULACACEAE}

Portulaca oleracea L., 0, T(M), QB 29, PC 50, PC 70, QB 19, PC 80.

Montia fontana L., R, M, PC 81, QC 00.

\section{CARYOPHYLLACEAE}

Arenaria montana L., 0, M, QB 09.

Arenaria conimbricensis Brot., R, M, QB 09

Arenaria conica Boiss., $\mathrm{Vr}, \mathrm{M}, Q B 28$.

Moerhingia pentandra Gay, R, M, QB 19.

Stellaria media (L.) Vill., A, T(M), QB 29, QB 09, QC 00, QB 18, PC 81.

Stellaria uliginosa Murray, $\mathrm{Vr}, \mathrm{T}(\mathrm{M}), \mathrm{PC} 81$.

Cerastium brachypetalum Pers., R, M, QB 09.

Cerastium glomeratum Thuill., A, T(M), QB 29, QB 18, QC 00, QB 09.

Cerastium fontanum Baumg., Vr, M, QB 09.

Moenchia erecta (L.) P. Gaertner, 0, $T(M), Q B$ 28, QB 18, QB 38, PC 70, PB 99.

Sagina apetala Ard., R, T(M), PB 99, PB 89, QC 00, QB 17.

Corrigiola telephiifolia Pourret, $A, T(M), Q B$ 29, QB 28, QB 18, QB 17, QB 19, PC 90, PB 99, PB 87.

Corrigiola litoralis L., $0, M(T), Q B$ 09, QB 08.

Paronichia cymosa (L.) DC., R, M, PB 99.

Paronychia echinulata Chater, $0, T(M)$, QB 29, PB 99, QB 19.

Paronychia argentea Lam., A, T(M), QC 00, QB 29, QB 09, QC 10, PB 99, QB 19.

Herniaria scabrida Boiss., Vr, M, QB 09.

Herniaria lusitanica Chandhri, 0, $T(M), Q B 09$, QB 19, QC 00.

Illecebrum verticillatum L., R, M, QB 29.

Polycarpon tetraphyllum (L.) L., A, T(M), QB 19, QB 09, PB 99, QB 29, PC 60, PC 00, PB 89.

Spergula arvensis L., F, T(M), QB 09, QB 18, PB 09 . 
Spergula pentandra L., Vr, M, QC 00, PC 01. Spergula morisonii Boreau, $\mathrm{Vr}, \mathrm{T}, Q B 27$.

Spergularia rubra (L.) J. \& C. Presl. A, $T(M)$, PC 70, PB 99, QB 09, QB 19, QB 29, QB 39, QC 00, PB 88.

Spergularia purpurea (Pers.) G. Bonfil., 0 , M, QC 00.

Spergularia bocconii (Scheele) Ascherson $\varepsilon$ Graebner, 0, M, QB 19.

Silene mellifera Boiss. \& Reuter, R, M, QB 09, QB 19.

Silene vulgaris (Moench) Garcke, F, T(M), QC 10, QB 19, QB 09, PB 99, PC 90.

Silene alba (Miller) Krause, A, T(M), QB 19, QB 09, QB 29.

Silene portensis L., $0, \mathrm{I}, \mathrm{PB} 87$.

Silene inaperta $L ., 0, T(\boldsymbol{H}), P B 29, P B 88, Q B$ 39, PC 70, QB 19.

Silene fuscata Link, R, M, PC 90.

Silene laeta (Aiton) Godron, Vr, T, QC 00.

Silene gallica L., $A, T(M), Q B 29, P B 99, Q B$ 19, QB 09, QC 00, QB 39, PC 81.

Silene scabriflora Bort., F, I(M), QB 09, QB 19, QB 29, PB 99.

Silene colorata Poiret, A, T(H), QB 29, QB 28, QB 39.

Silene secundiflora 0 tth, $0, \boldsymbol{M}, Q B \quad 09$.

Saponaria officinalis $L ., 0, I(M), Q B 09, P C$ 70, PC 90, PB 99, PB 88.

Petrorhagia nanteuillii (Burnet) Ball \& Heywood, F, I(H), QB 29, QB 19, QB 09, QC 00, QC 20, PB 99 .

Dianthus lusitanicus Brot., 0, T(M), PB 99 , QB 09, QC 00 .

Dianthus crassipes Roemer, R, I, QB 39.

Dianthus cintranus Boiss. \& Reuter, R, M, QB. 19.

\section{RANUNCULACEAE}

Nigella damascena L., $0, T(M), Q B 09, Q B 19$, QB 29.

Nigella hispanica L., O, T(M), QB 29.

Delphinium gracile DC., F, T(M), PB 99, QB 09, QB 19, QB 29, QB 39 .

Delphiniu staphisagria L., R, A, QB 09, PB 89.

Consolida ambigua (L.) Ball \& Heywood, R, M, QC 10.

Anewone palmata L., F, T(M), QB 28, QB 09, PB 99, QB 18, QB 19.

Clematis campaniflora Brot., F, M(I), QB 09, QC 00, QB 28, PC 70, PB 88, PB 89, PB 99.

Ranunculus gramineus L., $\mathrm{Vr}, \mathrm{M}, Q B 09$.

Ranunculus repens $L ., R, M, P B 99$.

Ranunculus bulbosus L., F, T(M), QB 09, PC 90, QC 00, PB 99, PB 59 .

Ranunculus muricatus L., F, M, PB 99, QB 29, QC 10.

Ranunculus arvensis L., 0, A, PB 89, QC 00.
Ranunculus parviflorus L., R, M, PB 89.

Ranunculus paludosus Poiret, $F, T(M), Q C 00$, QB 09, QB 28, QB 18, PC 70.

Ranunculus gregarius Brot., F, T(M), QB 09, QC 00, QB 28, QB 18, QB 19, PC 70.

Ranunculus ficaria L., F, M, QB 19, QB 09.

Ranunculus bullatus L., F, I(M), QB 39, QB 09, QB 19.

Ranunculus hederaceus $L ., 0, M, P C 80$.

Ranunculus tripartitus DC., $0, \mathrm{I}$, QB 39 , QC 00, QB 18.

Ranunculus ololeucos Lloyd, R, T, QB 49.

Ranunculus baudotii Godron, 0, I, PB 77, QB 49, PB 18.

Ranunculus trichophyllus Chaix, O, T, QB 39, PC 60 .

Thalictrum flavum L., O, T(M), QB 09, QB 19, QB 29, PC 80.

\section{PAEONIACEAE}

Paeonia broteroi Boiss. E Reuter, F, M, QB 09, QB 29, QC 00, PC 70.

\section{PAPAVERACEAE}

Papaver dubium L., R, M, PC 70.

Papaver hybridum L., R, H, PC 80.

Papaver rhoeas L., A, T(M), QB 09, QC 10, QB 19, QB 29, PC 90, QB 39.

Chelidonium majus L., $0, M(T), P C 90, P B 99$, PC 00, QB 09, PC 80.

Funaria agraria Lag., 0, I, QB 29, QB 39.

Fumaria capreolata L., A, I(M), QB 09, QC 1.0, QB 29, QC 00, QB 00, QB 19.

Fumaria bastardii Boreau, R, M, QB 09.

Funaria muralis Sonder, O, M, QB 09, QB 19.

Funaria reuteri Boiss., F, I(M), QB 19, QB 29, QB 09, QC 10, QC 00 .

Funaria officinalis L., A, $T(M), Q B$ 09, QC 00, QC 10 .

Funaria calcarata Cad., R, M, QB 19.

\section{CAPPARIDACEAE}

Cleone violaceae L., 0, T(M), PB 99, QB 09.

\section{CRUC IFERAE}

Alliaria petiolata (Bieb.) Cavara \& Grande, Vr, M, QC 00 .

Rhynchosinapis hispida (Cav.) Heywood subsp. - transtagana (Coutinho) Heywood, 0, M, QB 19, QB 29.

Arabidopsis thaliana (L.) Heynh., A, T(M), QB 19, QB 09, QC 00, PB 99, PC 90.

Hesperis laciniata All., Vr, M, QC 10.

Barbarea intermedia Boreau, R, H, QC 00, QB 19.

Barbarea vulgaris $R, B r ., V r, M, P B ~ 89$.

Nasturtium officinale $R$. $B r ., 0, T(H), P B 89$, QB 09, QB 29, PB 99, PC 90, QB 19, PC 70 .

Lunaria annua L., R, M, PB 99. 
Cardanine hirsuta L., F, T(M), QB 18, QB 19, QB 29, QB 39, PC 90, PB 99, QB 09.

Arabis stenocarpa Boiss. \& Reuter, R, M, QB 09. Alyssum minus (L.) Rothn., R, T, QB 39.

Alyssum granatense Boiss. E Reuter, 0, H, PB 09, QB 28, PB 99.

Draba muralis L., R, M, QB 09.

Erophila verna (L.) Chevall., R, M, QB 09.

Capsella rubella Reuter, $0, M(T), Q B$ 09, QC 00, PB 89, QB 19.

Capsella bursa-pastoris L., A, I(M), QB 09, QB 29, PC 80.

Hornungia petraea (L.) Reichenb., R, M, QB 09. Teesdalia coronopifolia (J.P.Berg.) Thell., F, $M(\mathrm{~T}), Q B$ 09, QB 29, QB 09, PB 99, PC 90, QB 19.

Thlaspi perfoliatum L., R, I, QB 39.

Iberis linifolia Loefl., F, M, QB 09, QB 19, QB 99, PC 80, QB 29.

Iberis saxatilis L., R, M, QB 29.

Biscutella laevigata L., R, M, QC 11.

Biscutella baetica Boiss. E Reuter, O, I, QB 38, QB 28, QB 39, QB 18.

Lepidium heterophyllum Bentham, 0, M, OC 10 , QC 00, QB 19, QB 09.

Cardaria draba (L.) Desv., R, M, QB 09.

Coronopus didymus (L.) Sm., R, H, QC 00, QB 29.

Crambe hispanica L., R, M, QB 09.

Diplotaxis catholica (L.) DC., O, T(M), QB 18, QB 29.

Diplotaxis muralis (L.) DC., F, T(M), QB 39, QB 29, QB 18.

Brassica nigra (L.) Koch, $F, M(T), Q B$ 09, QB 29, PC 00.

Brassica barrelieri (L.) Janka, F, I(M), QB 39, QC 00.

Brassica napus L., F, I(M), PC 00, PB 99, PC 90.

Sinapis alba L., 0, M(T), QC 00, QB 09, PC 70, QB 29.

Sinapis arvensis L., $0, M(T), Q B$ 09, QC 00, PC 90, QB 28.

Hirschfeldia incana (L.) Lagrèze-Fossat, $A$, $T(M), Q B 19, P C 70, Q B 39, Q B 29, P B$ 99, QB 09, PC 00.

Rapistrum rugosum (L.) All., 0, M, QC 10.

Calepina irregularis (Asso) Thell., 0, H, QB 09, PB 89, PC 00, QC 00, QC 90.

Rhaphanus raphanistrum $L ., A, I(M), P C 71, Q C$ $00, Q B$ 09, QB 29, QB 18, QB 10, QC 90.

\section{RESEDACEAE}

Reseda phyteuma L., F, T(M), QB 09, PB 99, QB 29, QB 19.

Reseda luteola L., F, T(M), $B B$ 09, QB 19, QB 00, QC 00, PC 70 .

Reseda media Lag., R, I, QB 88.

Sesamoides canescens (L.) O. Kuntze, A, T(M), QB 29, QB 09, QB 19, QC 00, PB 99.
CRASSULACEAE

Crassula tillaea Lester-Garland, $\mathrm{Vr}, \mathrm{T}, \mathrm{QB}$ 18.

Umbilicus rupestris (Salisb.). Dandy, F, $T(\boldsymbol{M}) \mathbf{r}$ PC 70, QB 29, QC 00, QB 09, QB 18, QC 10.

Unbilicus horizontalis (Guss) DC., R, M(I), QB 09, PB 99.

Mucizonia hispida (Lam.) A. Berger, 0, $M(\mathrm{I})$, QC 00, QB 19.

Sedun albun L., F, T(M), QB 28, QB 19, QB 09, PB 99, PC 90.

Sedun forsteranum Sm, F, M(T), PB 89, PC 80, QB 09, PB 99.

Sedum arenarium Brot., R, M, QB 29, PB 89.

Sedu brevifoliu DC., 0, $M(T), P B$ 99, QC 00.

Sedum caespitosu (Cav.) DC., R, I, QB 39.

Sedun tenuifolium (Sibth. \& Sm.) Strobl, $\mathbf{F}$, $\mathrm{I}(\mathrm{M})$, PC 81, QC 00, QB 19, QC 10, QB 29, PB $99, P C 80$.

Sedu sediforme (Jacq.) Pau, O, M(T), PB 99.

Sedum andegavense (DC.) Desv., R, M, QB 19, QB 28 .

\section{SAXIFRAGACEAE}

Saxifraga granulata L., F, M(T), QB 39, QB 09, PC 90, QB 28, QB 09, QC 00.

\section{ROSACEAE}

Rubus ulmifolius schott., A, M(T), PB 99, QB 29, QB 19, QC 10, QB 09 .

Rosa canina L., F, A, QC 10, QB 29, QC 20, PB 89.

Rosa pouzinii Tratt., F, M, QB 09, QB 29.

Rosa agrestis Savi, R, M, PB 99.

Agrimonia eupatoria L., F, M(T), QC 00, QB 19, PB 99, QB 09, PB 89.

Sanguisorba hybrida (L.) Nordborg, F, M, $Q B$ 19, QB 99, QB 09, PB 99, PB 59, QC 00, PC 81 .

Sanguisorba minor Scop., A, I(M), QB 09, QB 19, QC 00, QC 10, PB 99.

Geum sylvaticum Pourret, $\mathrm{Vr}, \mathbf{M}$, QB 09.

Geum urbanum L., Vr, M, QC 00.

Potentilla reptans L., 0, M(I), QB 09, QC 00, PC 90, QB 19, QB 29.

Fragaria vesca L., 0, M, QB 09, QB 19.

Aphanes cornucopioides Lag., R, M, QB 29, QB 19, QB 09 .

Aphanes microcarpa (Boiss. E Reuter) Rothm., 0, M, QB 09, QB 18.

Pyrus bourgaeana Decne, F, M(T), PB 99, QB 29, QB 39, QB 09, QB 19, PC 90.

Crataegus monogyna Jacq., F, I(M), PC 70, QB 09, QB 39, PC 80, PB 99.

\section{LEGUMINOSAE}

Cytisus baeticus (Webb) Stendel, F, M(T), PB 99, PC 80 , PB 99, PB 89.

Cytisus grandiflorus DC., 0, 月, PC 00, PB 99. 
Cytisus striatus (Hill) Rothm., A, M, PB 29, PC 80, PC 90, PB 89.

Genista falcata Brot., F, M(I), QB 09, QB 19, QC 00, PC 70.

Genista hirsuta Vahl., F, I(M), QC 10, QB 09, QB 19, PB 99, QB 29.

Genista triacanthos Brot., F, I(M), QB 09, QB QB 17, PC 90, PC 81.

Genista polyanthos R. Roemer ex Willk., F, I, PC 90, QB 28, QB 39.

Chanaespartium tridentatu* (L.) P. Gibbs, A, $M(T), Q B$ 09, QB 19, QC 00, PV 99.

Retama sphaerocarpa (L.) Boiss., 0, I, PB 99, PC 80, PC 70.

Spartium junceum L., 0, T(M), PC 00, $P C 80$.

Ulex eriocladus Vicioso, $A, T, Q B 29, Q B$ 09, PB 89, QB 39, QB 18, QB 17, PB 99.

Adenocarpus telonensis (Loisel.) DC., R, T, QB 29 .

Lupinus luteus L., 0, A, QB 09 PC 90.

Lupinus hispanicus Boiss. E Reuter, $\mathbf{0}, \mathbf{M}(\mathrm{I})$, QB 09 .

Lupinus angustifolius L., $F, T(M), Q C \quad 00, Q B$ 29, QB 09, QB 19, PC 90.

Lupinus micranthus Guss., R, M(T), QB 09, QB 29, PC 90.

Lupinus albus L., R, A, QB 19, QB 38.

Galega officinalis L., R, T, PB 59.

Astragalus lusitanicus Lam., $A, T(M), Q B 09$, QB 29, QB 19, QC 00, QB 39, PB 99.

Astragalus cymbicarpos Brot., R, A, PB 99.

Psoralea bituminosa L., A,T(H), QC 10, QB 09, QB 29, QB 19, PB 89, PC 80.

Vicia villosa Roth., 0, H, QB 09.

Vicia benghalensis L., A, $\boldsymbol{M ( T ) , ~ Q B ~ 2 9 , ~ Q C ~} 00$, QB 09, QB 19, PC 11.

Vicia disperma $D C ., F, T(M), P B 89, Q C 10, Q B$ 19, QC 00, PB 99, QB 09.

Vicia pubescens (DC.) Link, Vr, M, QB 09.

Vicia sativa L., A, T(M), QC 10, QB 09, QC 00, QB 19, QB 29, PC 90 .

Vicia lutea L., F, I(M), QC 10, QC 00, PB 99, QB 09.

Vicia hybrida L., Vr, $M, Q B 09$.

Lathyrus latifolius L., A, M, PC 90, PB 99, PC 80 .

Lathyrus tingitanus L., F, M, QC 10, QB 39 , QB 19, QB 09 .

Lathyrus cicera L., F, M(I), PC 00, QB 09, QC 00 , QB 19.

Lathyrus angulatus L., 0, M, PC 90, QB 28, PB 99, QB 09, QB 29.

Lathyrus clymenum L., A, M(T), QB 29, PC 80 , QB 09, QC 00, QC 10 .

Lathyrus aphaca L., F, M(I), QB 09, PC 90.

Ononis natrix L., O, T, PB 99, QB 19.

Ononis biflora Desf., R, M, QB 09.

Ononis cintrana Brot., $F, M(T), Q B 29, Q B$ 09, QC 00, PB 59.

Ononis speciosa Lag., Vr, T, QB 29.
Ononis spinosa L., F, I(M), QB 09, PB 99, QC 10, $Q B$ 19, $P C 90, Q B 39$.

Ononis difusa Ten., R, T, QB 18.

Ononis viscosa L., $\mathbf{0}, \mathbf{M}, Q B 09$.

Ononis repens L., R, T, QB 29.

Biserrula pelecinus L., $0, T(M), P B 79, P C 80$,

Melilotus indica (L.) All., R, T(M), QB 09, QB 19 .

Melilotus segetalis (Brot.) Ser., R, M, QB 19.

Medicago disciformis DC., R, M, QB 19.

Medicago lupulina L., F, T(M), QB 29, QB 09, PC 90, QC 00, QB 19.

Medicago sativa L., F, T(M), QB 19, QB 09.

Medicago orbicularis (L.) Bartal, R, M, PB 99,

Medicago tornata (L.) Miller, R, M, QB 19.

Medicago truncatula Gaertner, $0, T(M), Q B 19$, PB 99.

Medicago aculeata Gaertner, 0, T, QB 19, QC 00, QB 39.

Medicago polymorpha L., A, $\boldsymbol{N ( T ) , P C ~ 9 0 , ~ Q C ~ 0 0 , ~}$ QB 29, QB 09, QC 10.

Medicago arabica (L.) Hudson, $0, T(M), Q C 00$, QB 19.

Medicago minima (L.) Bartal, R, M, QB 19.

Irifolium repens L., A, M(T), QB 19, QB 09, QC 10, PB 99, PC 90, PB 89.

Trifoliun glomeratum L., $A, T(M), Q B 19, P B$ 89, QC 10, QB 09, PB 99, QC 00.

Irifoliun suffocatun L., R, A, PB 99.

Trifolium resupinatum L., R, M, QB 29.

Trifoliun tomentosu $L ., A, M(T), Q C 00, Q B$ 09, PB 99, PC 90, QB 29.

Trifoliun campestre Schreber, A, M(T), QB 19 , QB 29, PB 89, QC 10, PC 90.

Irifolium striatum L., R, T, QB 39.

Trifolium arvense L., A, T(M), QB 29, QB 09, QB 19, PB 99, QB 17.

Irifolium bocconei Sari, Vr, M, QB 18.

Trifoliun gemelum Pourret ex Willd., R, T, QB 19 .

Trifoliun ligusticun Balbis ex Loisel, VR, I, QC 00.

Trifolium scabrun L., F, T(M), PB 99, QB 29.

Trifolium stellatum L., A, T(M), PC 90, QC 00, $Q B$ 19, $Q B$ 09, QC 10, $Q C$ 00, $P B$ 99, $P C$ 80 .

Trifolium pratense L., F, M(I), PB 99, QB 29, QB 09.

Trifoliun lappaceun L., R, I, QB 00 .

Trifoliun cherleri L., 0, I(M), QB 09, QB 29.

Trifolium angustifolium L., $A, T(M), Q B 19$, QC 10, QB 19, QB 29, QC 00, PB 89.

Trifoliun subterraneu $L ., F, T(M), Q B 18, Q C$ 10, QB 19, PC 90.

Dorycnium rectum (L.) Ser., $0, M, Q B 29, P B$ 89, QB 09, PB 99.

Dorycnium pentaphyllum Scop., R, M, QB 29.

Lotus uliginosus Schkubr., F, M(T), PB 99, QB 29, QB 19, QB 09, PC 80.

Lotus castellanus Boiss. E Reuter, F, T(M), 
QB 28, $P B$ 88, QC 10, QB 38, PB 58.

Lotus parviflorus Desf., F, T(M), PB 50, PB 81, PC 90, PC 10, QB 39, PC 00.

Lotus conimbricensis Brot., $0, M(I), Q C 00$, QB 17, PC 90.

Anthyllis gerardi L., $F, M(T), Q B$ 19, QB 29, QC 10, PB 99, QB 29, PC 90, QB 09.

Anthyllis vulneraria L., F, M(T), QB 09, QC 00, QB 09, PB 99.

Anthyllis lotoides L., F, M(T), PC 90, PB 99, PC 70, QB 29, QC 10.

Anthyllis cornicina L., Vr, H, QB 29.

Ornithopus compressus $L ., A, M(T), Q C 10, P B$ 89, QC 00, QB 29, QB 09, PB 99, PC 90, PC 81 .

Ornithopus pinnatus (Miller) Druce, 0, M, QC $00, P B 89$.

Coronilla juncea L., 0, M(T), QB 19, QB 29.

Coronilla valentina $L$. subsp. glauca (L.) Batt., R, M, PC 90, QB 09.

Coronilla repanda (Poiret) Guss. subsp. dura (Cav.) Coutinho, F, M(I), QB 29, QB 09, PB 89, PC 10, PC 81 .

Hippocrepis scabra DC., R, A, QB 09, QB 29.

Scorpiurus muricatus L., $F$, M(T), QB 19, QB 29, QC 00, QB 19, PB 99.

Scorpiurus sulcatus L., R, M, QB 19.

Scorpiurus verniculatus L., F, T(M), QB 19, QB 09, PC 90, QB 28.

\section{OXAL IDACEAE}

0xal is corniculata L., F, N(T), QC 00, QB 09, PC 90, PB 99.

Oxalis latifolia Kunth, R, M, QC 00, QB 09.

0xalis pes-caprae L., $F, M(T), Q B 28, Q B 09$, QB 29.

\section{GERANIACEAE}

Geranium columbinum L., R, M, PB 89.

Geranium rotundifolium L., F, T(M), QC 00 , QB 29, QB 09, QB 28, QB 18, QB 39, PB 89.

Geranium molle L., $A, M(T), Q B$ 09, $P B$ 99, QC 00, PC 70.

Geranium dissectum L., F, M(T), QB 29, QC 00, QB 19, QB 09, PB 58.

Geranium lucidum L., F, M(I), QB 29, QC 00, QB 09, QB 19, PB 99.

Geranium robertianum L., 0, M, QB 09, PB 99. Geranium purpureum Vill., A, M(T), QB 09, QB 19, QC 10, QC 00, PC 81, PC 80, PC 90 .

Erodium malacoides (L.) L'Hér., R, M, QC 00.

Erodium botrys (Cav.) Bertol., F, M(I), QB 17, $Q B$ 29, QB 09, QB 38, QB 27, QB 19.

Erodium cicutarium (L.) L'Hér., $0, T(N), Q B$ 39, QC 00, QB 09, QB 18.

Erodium moschatum (L.) L'Hér., F, M(I), QB 18, QC 00, QB 09, QB 39, QB 29.

\section{LINACEAE}

Linum tenue Desf., F, M(I), QB 09, QB 19, PB 99, QB 29.

Linum strictum L., 0, T(M), PB 99, QB 29, QB 19.

Linum bienne Miller, A, I(M), QB 19, PB 99, QC 10, QC 00 .

Linum trigynum L., 0 , M(I), QB 29, QB 09, PB 58.

Radiola linoides Roth, 0, M(T), QB 09, QC 10, QB 29, PB 59, PB 88.

\section{EUPHORBIACEAE}

Securinega tinctoria (L.) Rothm., 0, T, PB 99, QB 39.

Mercurialis annua L., A, I(M), QB 29.

Euphorbia chamaesyce L., 0, I(M), QB 29, PC 50, PC 60 .

Euphorbia pubescens Vahl, F, M, PC 90, QB 19, QB 09 .

Euphorbia helioscopia L., F, M(T), QB 29, QB 09, QB 39, PC 90, PB 89.

Euphorbia exigua L., A, T(A), PC 90, PB 99, QB 09, PB 89, QB 19, QB 29, PC 70.

Euphorbia falcata L., R, A, QB 29, QB 39.

Euphorbia peplus L., F, M(T), QB 18, PC 90 , QB 19, QB 28, QB 09 .

Euphorbia segetalis L., O, M, QB 19, QB 09.

Euphorbia pinea L., O, M, QB 19, QB 29.

Euphorbia nicaensis All., F, M, PC 10. QB 19, QB 09.

Euphorbia esula L., 0, M, QB 09, QB 19, QB 39.

Euphorbia amaygdaloides L., 0, M, QB 09.

Euphorbia characias L., F, M(I), PB 99, PC 00, QB 09, PC 90, QB 29.

\section{RUTACEAE}

Ruta montana (L.), L., F, T(M), QC 10, QB 19, PC 90, QB 29, PB 99.

Ruta angustifolia Pers., 0, $T(M), Q B$ 09, QB 19, PB 99.

Ruta chapelensis L., R, $M(T), Q B 09$.

\section{POLYGALACEAE}

Polygala microphylla L., R, A, QB 09. Polygala monspeliaca L., R, M, QB 29.

\section{ANACARDIACEAE}

Rhus coriaria L., R, M, QB 09, QB 19.

Pistacia terebinthus L., F, M(T), QB 09, QC 00, PB 99, QB 29.

Pistacia lentiscus L., A, T, QB 09, PC 70.

\section{RHAMNACEAE}

Rhamnus alaternus L., $F, M(T), Q B$ 09, $P B 99$, PC 70, QC 00.

Rhannus lycioides, L. subsp. oleoides (L.) Jahandiez \& Maire, 0, I, QB 09, QB 19, QB 29. 
Frangula alnus Miller, R, M, QB 09.

\section{MALVACEAE}

Malva hispanica L., A, T(M), PB 99, PC 80 , PB 58, QB 19, PC 81.

Malva tournefortiana L., $0, \mathrm{~A}$, QC 00.

Malva sylvestris L., A, M(T), QB 29, QB 19, PB 99, QC 00 .

Malva nicaensis All., R, H, QB 19.

Lavatera cretica L., F, $M(T), P C 80, Q B$ 09, PB 99.

Alcea pallida (Willd.) Waldst. E Kit., 0, M, QB 19, QB 09, QC 00.

\section{THYMELAEACEAE}

Daphne gnidium L., A, T(M), QB 09, PB 89, PB 99, QC 00, PB 59.

Thymelaea villosa (L.) Endl., F, M(T), QB 09, PB 99, QB 19, PB 59, PC 90.

\section{GUTTIFERAE}

Hypericun humifusum L., $0, M(T), Q B 29, \quad Q C$ $00, P B 89, P C 81, P C 50$.

Hypericum perforatum L., A, T(M), QB 19, $P B$ 99, QB 29, QB 09, QC 00, QC 10.

Hypericum undulatum Schousboe ex Willd., F; $M(T), Q C$ 00, QB 09, PB 99, QB 29, PB 89 , PB 58.

Viola odorata L., F, M(I), QB 39, QB 09, QB 19.

Viola riviniana Reicenb., F, M, QB 09, PC 00, PB 99, QC 00, QB 19.

Viola arvensis Murray, F, $\mathbf{I}(\mathbf{M})$, QB 19, PB 89 , QB 19, QB 09, QC 00, PC 00.

Viola kitaibeliana Schultes, Vr, T, QB 39.

Viola demetria Prolongo ex Boiss., R, M, $Q B$ 09 .

\section{CISTACEAE}

Cistus cripus L., 0, I(M), PB 99, QB 29, QC 10, QB 09, QB 19, PC 81 .

Cistus albidus L., $0, T(\boldsymbol{H}), Q B$ 09, QB 19, QB 29.

Cistus monspeliensis L., A, T, QB 28, PB 99 , QB 09, PB 89.

Cistus psilosepalus Sweet, $\mathrm{Vr}, \mathrm{T}, \mathrm{PB} 99$.

Cistus salvifolius L., A, I(M), QB 09, QC 00, QB 29, PB 99, QB 19.

Cistus populifolius L., $F, M(T), P B$ 99, QB 09, QB 29, PC 81, QC 00, QB 19.

Cistus ladanifer L., A, T(N), PB 99, QC 00, QB 09, QB 29, PC 81.

Haliøium ocymoides (Lam.) Willk., $F, M(T), Q B$ 19, PB 99, QC 00, QB 09, QC 10, PC $81, P B 88$.

Halinium lasianthum (Lam.) Spach., R, H, QB 09.

Haliøium haliøifolium (L.) Willk. R, T, QB 39.
Halimium viscosum (Willk.) P. Silva, R, I, QB 38, QB 19, PC 70, QB 29.

Tuberaria lignosa (Sweet) Samp., F, M, QC 00, QB 29, QB 09, PC 80.

Tuberaria guttata (L.) Fourr., A, T(M), QB 19, QB 09, QB 29, QC 00, PC 81.

Tuberaria macrosepala (Cosson) Willk. Vr, I (M), QB 19, QB 29.

Helianthemum ledifolium (L.) Miller, R, T(M), QB 09, PB 99.

Helianthemum salicifolium (L.) Miller, 0, M, QB 09.

Helianthemum sangineum (Lag.) Lag. ex Dunal, $R, T, Q B 37$.

Helianthemum aegyptiacum (L.) Mill., 0, T(M), QB 28, QB 39, QB 29.

\section{TAMARICACEAE}

Tawarix gallica L., R, T, PC 50 .

\section{CUCURBITACEAE}

Bryonia cretica L. subsp. dioica (Jacq.) Tutin, $F, T(M), P C 81, P C 70, Q B 39, Q C$ $00, Q B 29, Q C 10$.

\section{LYTHARACEAE}

Lythrum salicaria L., 0, M(T), QB 19, PC 90, QB 29, PC 90, QB 09, QC 00.

Lytrum junceum Banks. E Solander, $F, T(\boldsymbol{M}), Q B$ 29, QB 09, QB 39, PB 89, QB 19.

Lythrum hissopifolia L., 0 , M(I), QB 29, PB 87, QB 09, QC 00, PC 81, QB 19.

Lythrum borysthenicum (Schrank) Litv., R, T, PB 87.

Lythrum portula (L.) D.A. Webb, R, T, PB 59, PC 81, PC 60 .

\section{MYRTACEAE}

Myrtus commis L., F, I(M), QB 39, QB 38, QB 18, PB 99, QC 00, QB 09.

\section{ONAGRACEAE}

Epilobiun hirsutun L., F, M(T), PC 90, QB 19 , QC 00, PC 60.

Epilobiu parvifloru Schreber, $0, M(T), Q B$ 09, PB 99.

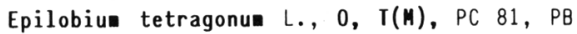
88, QC 00.

Epilobiu tetragonun L., subsp. tournefortii (Michalet) Léveillé, 0, I, QB 29.

Epilobium obscurum Schreber, R; ', QB 09.

HALORAGACEAE

Myriophyllum spicatu凶 L., R, T, QC 60, QB 39. ARAL I ACEAE

Hedera helix L., 0, M(I), QB 29, PB 99, QB 09, PC 90. 


\section{UMBELLIFERAE}

Eryngium campestre L., F, T(M), QB 39, QB 29, $P C$ 50, PC 70, PB 99, PC 90, QB 19, PC 00 .

Anthriscus caucalis Bieb., R, M, QB 09.

Scandix pecten-veneris L., $F, M(T), P B 99, Q C$ 00, QB 18, QB 09, QB 19, PB 89.

Smyrnium olosatrum L., $0, \mathbf{M}(\mathrm{T}), \mathrm{HC} 70, \mathrm{PC} 80$, QB 39, PB 89, PC 00.

Conopodium majus (Gouan) Loret., subsp. ranossum (Costa) S. Silvestre, F, M(I), QB 09, PB 99, PC 81, QB 39, QC 10, PB 89.

Pimpinella villosa Schousboe, 0, M(T), PB 99, QB 29, PC 90, QB 19.

0enanthe crocata L., 0 , M(I), PC 81, QB 29, QB 19, QB 09, PB 99, PB 58.

Foeniculum vulgare Miller subsp. piperitum (Ucria) Coutinho, A, T(M), PB 99, QC 10, QB 19, QB 09, PC 60, QB 29, PC 80.

Conium maculatum L., R, T(H), QB 29, PC 00.

Magydaris panacifolia (Vahl) Lange, $\mathbf{0}, \mathbf{M ( I )}$, PB 99, QB 19, PC 80.

Bupleuru fruticosun L., 0, M, QB 19, QB 09. Bupleurun gibraltaricum Lam., R, M, Qb 19.

Apiun nodiflorun (L.) Lag., $0, M(I), P C 00$, QB 29, QC 10, QB 09, PC 70, QB 39, PC 81 .

Ridolfia segetum Moris, R, M, QB 19.

Ferula comonis $1 ., 0, M(T), Q B 39, P B 99, Q B$ 09 .

Ferulago brachyloba Boiss., $\mathrm{Vr}, \mathrm{T}(\boldsymbol{M}), \mathrm{PB} 99$.

Elaeoselinu foetidu (L.) Boiss., F, M(T), PC 90, PB 99, QB 09, QB 19, QC 00.

Elaeoselinu gumaiferua (Desf.) Tutin, R, M, QB 19 .

Thapsia villosa L., A, M(I), QB 09, QC 10, QB 19, QB 29, QC 00, PC 81.

Thapsia maxima Miller, F, H, $Q B$ 29, $P B$ 89, QB 09, PC 80

Thapsia garganica L., R, T(M), PC 70, QB 17.

Torilis nodosa (L.) Gaertner, $0, M(T), Q B$ 09, QB 29, QC 10, PB 99.

Torilis arvensis (Hadson) Link, A, M(I), QB 09, QC 10, QB 09, PB 99, QC 00.

Orlaya kochii Heywood, 0, M(T), QB 19, QB 09, PB 99, PB 89.

Daucus durieua Lange, R, T, QB 19.

Daucus crinitus Desf., F, M(T), QB 19, QB 09, $P B$ 99, QB 29, QB 18, QB 39.

Daucus carota L., F, T(M), QB 19, PB 99, QB 09, QB 29, QC 10, PC 70.

\section{ERICACEAE}

Erica andevalensis Cabezudo \& Rivera, R,M(T), QB 07, QB 08, PB 88, QB 17, PB 66, PB 78.

Erica umbellata L., F, M, QB 29, PC 00, QB 09, QB 19, QC 10 .
Erica australis L., A, M(I), QC 10, QB 19, QB 29, PC 00, QB 18, PB 58.

Erica arborea L., $F, M(I), Q C$ 19, QB 09, QB 18, PB 89, QB 19.

Erica lusitanica Rudolphi, 0, M, $P B$ 89, QB 18, QB 27, QC 10, QB 38, Qb 39, PB 99.

Erica scoparia L., F, M(T), PB 99, QB 18, PB 38, QB 29, QB 17, QB 19.

Calluna vulgaris (L.) Hull, R, T, PB 66.

Arbutus unedo L., F, H, QB 19, QB 09, QC 00, PB 87.

PRIMULACEAE

Primula vulgaris Hudson, 0, M, QB 09, QC 00, PC 90, PB 99.

Asterolinon linum-stellatu (L.) Duby, $0, T(M)$, QB 09, PC 81 .

Anagallis tenella (L.) L., R, M, PC 90, PB 84 , PC 90, QC 10.

Anagallis arvensis $L ., \sigma^{\circ}, \mathbf{M}(\mathrm{T}), P C 90, Q B 19$, $P B$ 99, QC 10, QC 00, QB 29, QB 09.

Anagallis monelli L., F, T(M), QB 29, $Q B$ 09, PB 99, PC 90.

Samolus valerandi L., R, M, QB 09, QB 19, PB 99.

\section{PLUMBAGINACEAE}

Armeria littoralis Willd., 0, T(M), QB 39, PB 99.

Limonium sinuatum (L.) Miller, R, I, PB 87.

OLEACEAE

Jasminum fruticans L., R, M, QB 09.

Fraxinus excelsior L., $0, M(T), P B$ 99, PC 00, QB 39.

Olea europaea L., var. sylvestris Brot., F, $T(\boldsymbol{M})$, PB 99, QB 39, PC 70.

Phillyrea angustifolia L., F, T(M), QB 09, QB 19, QC 10, PB 99, PC 90.

Phillyrea latifolia L., F, M(T), QB 19, QC OO, QB 09 .

\section{GENCIANACEAE}

Blackstonia perfoliata (L.) Hudson, R, T(M), QB 29, PB 87.

Centaurium erythraea Rafn, $F, M(T), Q B 19$, $P B$ 99, QB 09, PC 81.

Centaurium maritimu (L.) Fritsch, R, M(T), PB 89, QB 29, PC 58.

\section{APOCYNACEAE}

Nerium oleander L., $0, T(M), Q B$ 09, PC 80, PB 99, QB 19, QB 18, PB 89.

Vinca difformis Pourret, $0, M(I), Q B$ 09, QB 39 , PC 70, PB 99.

Vinca major L., 0, $(\mathrm{I}), Q B$ 19, PB 99.

\section{ASCLEPIADACEAE}

Vincentoxicum nigru» (L.) Moench., O, M, QB $09, P B 99$. 


\section{RUBIACEAE}

Sherardia arvensis L., A, M(T), QC 00, QB 09, QC 10, PB 89, QB 19.

Crucianella angustifolia L., F, M(I), PC 81 , QC 00, PB 99, QB 19, QB 29.

Galiua aparine L., F, $M(T), Q B$ 09, QB 29, QC 00.

Galiu tricornutum Dandy R, M, QC 00.

Galium verrucosum Hudson, $F, M(T), P B$ 99, QB 39, QB 19, QC 00.

Galiu parisiense L., $F, M(T), Q B 29, P C 81$, QB 19 .

Galium murale (L.) All., R, M, QB 09, QB 29, QB 19.

Galium broterianum Boiss. \& Reuter, R, M, OC 00 .

Galium uliginosun L., R, M, QB 09.

Galium verum L., $0, M, Q B 19$.

Galium mollugo L., R, A, QB 19.

Galium spurium L., R, A, QB 19.

Rubia peregrina L., $F, M(T), Q B$ 09, $P B 99$, QC 10, QC 00, QB 29.

\section{CONVOLVULUS}

Cuscuta epithymum (L.) L., 0, $(T), P B 99$, PB 89, QB 19.

Cuscuta planiflora Ten., R, M, QB 19.

Calystegia sepium (L.) R. Br., R, H, QC 00, QB 09.

Convolvulus siculus L., R, M(T), QB 19, PC 81, QC 00, QB 09, PB 99.

Convolvulus althaeoides L., F, $M(T), Q B 29$, QB 09, PC 90, PB 99.

\section{BORAGINACEAE}

Heliotropiun europaeun L., F, M(I), QB 19, QB 29.

Neatostema apulum (L.) J.M. Johnston, R, I(M), PB 99, QB 29.

Cerinthe major L., R, A, QB 19.

Echiu plantagineu $L ., \quad A, M(T), Q B 29, \quad Q C$ $00, \quad Q B$ 17, QB 09, OC 10, PB 99, QB 19.

Echium creticum L., R, M, QB 09.

Echiu tuberculatu Hoff. \& Link, $F, M(I)$, QB 29, QB 09, QB 19.

Echiun rosulatun Lange, $F, M(T), P B$ 99, QB 19, PC 00, PC 10.

Nonea vesicaria (L.) Reichenb., O, I, QB 39.

Anchusa azurea Miller, F, M(T), PB 99, QB 19, QB 29, QB 09, QC 00, PC 99.

Anchusa undulata L., F, M(T), QB 19, QB 09, QC 00 , PB 99.

Anchusa undulata L., subsp. viciosoi Valdés, $0, H(T), Q B 19, P B 99$.

Borago officinalis L., $0, M(T), Q B$ 09, $Q B 29$, QC $00, P C 90$.

Myosotis debilis Pomel, R, A, QB 09, QB 19.

Myosotis secunda A. Murray, 0, M(I), PC 81 , QC 00, PB 89.
Myosotis welwitschii Bois's. \& Reuter, R, M, PB 99.

Myosotis discolor Pers., 0, M(I), PC 80, QC 00, QB 09.

Myosotis sicula Guss., R, T(N), QB 29.

Myosotis ramosissima Rochel, F, M(I), QC 00, QB 09, QB 29, PC 70, PB 99.

Omphalodes linifolia (L.) Moench, R, M, QB 09. Cynoglosu creticum Miller, F, M(T), QB 09, PB 99, QC 00, PC 90.

\section{VERBENACEAE}

Verbena officinalis L., $F, M(T), Q B$ 19, PB 99, QB 09.

Verbena supina L., R, M, QB 19.

\section{CALL I TRICACEAE}

Callitriche stagnalis Scop., 0, M(I), PB 99, PC 81 .

Callitriche obtusangula Gall, R, T(M), PC 81 . Callitriche platycarpa Kutz., R, I(M), QC 00. Callitriche palustris L., F, M(I), QB 09, QB 39, QB 19, PC 90 .

\section{LABIATAE}

Ajuga iva (L.) Schreber, O, T(H), QB 29, PB 99, QB 19.

Teucrium fruticans L., $F, N(T), Q B 09, Q B 19$, PB 99.

Teucrium capitatus (L.) Argangeli, 0, A, QB 19, QB 29.

Teucrium scorodonia L., F, M, PB 99, QB 09, QC 00, PB 89.

Teucriun polium L., O, M(T), PC 90, QB 29.

Marrubium vulgare L., F, M(T), QB 09, QB 19, QB 29, QC 10, PB 99, QC 00.

Sideritis arborescens Salzm. ex Benth., $\mathbf{0}$, M(T), QB 29, QB 19.

Phlonis purpurea L., F, T(H), PB 99, QC 00, QB 09, QC 10, QB 29, QB 39.

Phlowis lychnitis L., R, T, PB 99.

Laniun amplexicaule L., A, I(N), QB 29, PC 00, QB 09, QB 39, QC 00, PC 90.

Lanium purpureum L., R, A, QC 00, PC 90.

Stachys arvensis (L.) L., F, I(M), QB 09, PC $90, Q B 19, Q B 38, Q C 00$.

Stachys annua (L.) L., R, M(T), QB 09.

Stachys germanica L., $F, M(T), P B$ 99, Qb 39, PC 80, QB 29, QB 19.

Nepeta multibracteata Desf., R, I(M), PC 81 , PB 56.

Prunella vulgaris L., F, M, QC 10, QB 09, QC 00, PC 80, PC 81, PB 99.

Prunella laciniata (L.) L., R, M, PC 90, QB 09.

Melisa officinalis L., F, $M, Q B$ 19, PB 99, PC 80, QB 09 .

Calanintha sylvatica Bromf. subsp. ascendens (Jordan) Ball, A, M(T), QB 09, Qb 19, PC 90, PB 99. 
Clinopodiun vulgare L., F, H, QB 09, QB 29, QC 10, PB 99, PC 81.

Microneria graeca (L.) Reichenb., F, M(T), QB 09, QB 19, QB 29, PB 99.

Origanu凶 virens Hoff. \& Link, $F, M(T), Q B$ 19, QC 00, PC 90, PB 99, QB 09, QB 29.

Thymus mastichina L., $F, \mathbf{I}(\boldsymbol{M})$, QB 29, QB 19, QB 09, PB 99.

Mentha pulegiun L., 0, T(H), PB 99, PC 90, QB 29, PC 90, PC 60, PB 59.

Mentha spicata L., $\mathbf{0}, \mathbf{M}, P C 90$.

Mentha suaveolens Ehrh, A, M(T), QB 09, QB 90, QB 19, PB 89, PC 90.

Rosmarinus officinalis L., F, T(H), QB 18 , QB 39, QB 09, QB 29, QB 28.

Lavandula stoechas $L$. subsp. sanpaina Rozei$\mathrm{ra}, \mathbf{F}, \mathbf{I}(\mathbf{M})$, QB 39, QC 10, QB 18, QB 09, QB 29, PB 99, QC 00.

Lavandula stoechas L. subsp. luisieri (Rozeira) Rozeira, F, T(M), QB 19, PC 81, $P C 90, Q B$ 09, QC 00, QB 28.

Lavandula viridis L'Hér., R, T(M), QB 19, PB 58.

Salvia verbenaca $L$., $A, T(\boldsymbol{M}), Q B$ 19, QB 29 , QB 18, QC 00, PB 99, QB 09, QB 38.

\section{SOLANACEAE}

Hyoscyanus albus L., R, T, QB 39.

Solanun nigrun L., F, T(M), QB 39, PC 90, QB 29, QB 19, PB 99.

Solanum dulcamara L., R, A, PC 90, PB 89.

Datura stramoniun L., $0, T(M), P C 90, Q B 29$, PC 70, PC 80, PB 88, QB 19.

SCROPHULARIACEAE

Verbascum sinuatu凶 L., F, M(I), QB 19, QB 09, PB 99, QB 29, PB 89, QB 39.

Verbascum virgatum Stokes, $F, T(M), Q C 10$, QC 00, QB 09, PB 89, QB 19, PB 99.

Scrophularia canina L., F, M(I), QB 29, PB 89, QB 19, QB 09, PC 80.

Scrophularia scorodonia L., 0, M, PB 99, QB 29, QC 10, QB 09.

Scrophularia auriculata L., $0, M(T), Q C 00$, PB 99, QB 29, QB 39, QB 09.

Anarrhinu bellidifoliun ( $L$.$) Willd., A, T(M)$, PB 99, QC 10, PB 89, QB 29, PC 81,

Antirrhinum meonanthu Hoff. $\&$ Link. subsp. onubensis Fernandez Casas, $0, M, Q B$ 09, QB 29, PB 99.

Misopates orontiun (L.) Rafin, $A, M(T), Q B$ 19, QB 28, QC 00, QB 00, PB 99.

Chaenorhinu. villosuø (L.) Lange, O, M, QB 09, PB 99.

Linaria spartea (L.) Willd., R, T(M), QB 28, QC 10.

Linaria oblongifolia (Boiss.) Boiss. E Reuter subsp. hanseleri Valdés, $V r, M, Q B$ 09.

Linaria onubensis Pau, $\mathrm{Vr}, \mathrm{T}, \mathrm{QB} 17$.
Linaria saxatilis (L.) Chaz. R, M, QC 00.

Cymbalaria muralis P. Gaertner, 0, M, PC 90. QC 00, QB 09.

Kickxia cirrhosa (L.) Fritsch, Vr, T, PB 88 ,

Kickxia elatine (L.) Dumort., F, M(T), QB 19, QC 00, PB 89, PC 90.

Digitalis purpurea L. subsp. mariana (Boiss.) R. Goday, F, M, QB 09, QB 19, QC 10, PB 99, PC 81.

Veronica anagallis-aquatica L., $0, M(T), Q B$ 29, PB 99, QB 19, PC 80, PC 81.

Veronica persica Poiret, $F, M(I)$, QC 00, PB 99, QB 29, QB 09.

Veronica hederifolia L., F, M(I), QB 09, QB 39 , PC 80, PC 90, PC 00.

Veronica arvensis L., $\mathbf{0}, \mathbf{M}(\mathrm{I}), Q B$ 09, PC 90, QB 29.

Parentucelia latifolia (L.) Caruel, $0, M(T)$, QB 19, QB 29, QB 09, PC 70, PC 80.

Parentucelia viscosa (L.) Caruel, 0, I(M), QC 00, QB 29, QB 39.

Bellardia trixago (L.) All., 0, M, QB 29, QB 09, PB 89.

\section{ACANTHACEAE}

Acanthus mollis L., $0, M(T), Q B$ 09, QB 39, PC 80 , PB 99.

\section{OROBANCHACEAE}

Orobanche crenata Forskal, 0, M, QB 09, PB 99. Orobanche alba Willd., Vr, M, QB 19.

Orobanche ramosa L., 0, M, PB 89, QB 19, QB 09.

Orobanche foetida Poiret, R, M, QB 19.

Orobanche rapum-genistae Thuill., R, M, PB 99.

Orobanche sanguinea $C$. Presl, $0, M, P B 89, P C$ 90 .

LENT IBULARIACEAE

Pinguicula lusitanica L., R, T, PB 78.

\section{PLANTAGINACEAE}

Plantago ajor L., F, M(T), PB 99, PC 90, QC 10, PB 89, QB 19, QB 09 .

Plantago coronopus L., $F, T(M), Q B 29, Q B 28$, QB 19, QB 09, QC 10, PB 99.

Plantago serraria L., R, H, QB 29.

Plantago lanceolata L., A, M(I), QB 19, QB 39, PB 99, QC 00, PC 90.

Plantago lagopus L., F, M(T), PB 99, QB 19, QB 29, QB 39, QC 00, QC 10, QB 09.

Plantago bellardi All., 0, M(I), PB 99, QB 29. Plantago afra L., A, I(M), QC 10, PB 99, QB 09, QB 29, PC 70, QB 19.

\section{CAPR IF OLIACEAE}

Sambucus nigra L., 0, M, PB 89, PC 90, PB 99, QB $09, Q B 19$.

Viburnu凶 tinus L., F, M, PC 90, QC 00, QB 09, PB 99 . 
Lonicera periclymenum L., $F, M(T), Q C$ 00, QC 10, QB 19, PB 99, QB 09, PB 88.

Lonicera implexa Aiton, $F, M(T), P C 90, Q B$ 19, PB 99, QC 00.

\section{VALERIANACEAE}

Valerianella locusta (L.) Laterrade, R, M, QC 00 .

Valerianella microcarpa Desv., $F, M(T), Q B$ 28, QB 29, PB 99.

Valerianella carinata Loisel., F, M(T), QC 00, QB 18, QB 38, QB 19.

Valerianella coronata (L.) DC., O, I(M), QB 18, QB 09, QB 19, QB 29, QC 00.

Centhranthus calcitrapae (L.) Dufresne, F, $M(T)$, QB 29, QB 09, QB 19, QC 10, QC 00 .

Centhranthus calcitrapae (L.) Dufresne subsp. trichocarpus Richardson, R, H, QB 29.

\section{DIPSACACEAE}

Dipsacus comosus Hoff. E Link, R, M, QC 00.

Dipsacus fullonum L., $R, T(M), Q C 00, P C 60$. Dipsacus sativus (L.) Hinckeny, R, M, PB 99.

Scabiosa atropurpurea L., A, M(T), QB 19, QB 09, PB 99, PC 70, QB 19.

Scabiosa simplex Desf. subsp. dentata (Jordan E Fourr.) Devesa, 0, M(T), PB 99, QB 09, QB 29, PC 90 .

\section{CAMPANULACEAE}

Campanula primulifolia Brot. 0, M, QC 00, PB 89.

Campanula erinus L., F, T(M), QB 09, QB 19, PB 99, PB 89, QC 10, QB 29.

Campanula lusitanica L., $F, M(T), Q C \quad 00, Q B$ 09, PB 58, PC 81, PB 99.

Campanula rapunculus L., $A, M(T), Q B$ 09, QB 19, PB 99.

Legousia castellana (Lange) Samp., $R, T(M)$, PB 79.

Tracheliun caeruleu凶 L., $0, M(T)$, QC 00, PC 90.

Jasione montana L., R, $T(M), Q B$ 09, PB 58.

Jasione montana $L$. subsp. echinata (Boiss. $\&$ Reuter) R. Martínez, $F, M(I), P C$ 81, QB 19, PB 99, PB 88, QB 29, QB 28, QC 10 .

Lobelia urens $1 ., 0$, M, QC 00.

Laurentia gasparrinii ( $T$ ineo) Strobl, $0, T(M)$, PB 88, PB 59, QB 39.

\section{COMPOSITAE}

Bellis annua L., A, T(M), QB 28, QB 29, QB 18, QB 39.

Bellis perennis L., $F, M(T), Q B$ 09, PC 90.

Bellis sylvestris Cyr., A, M(T), QB 09, QC 00, QB 19, QB 18, PB 99, QB 39.

Conyza canadensis ( $L$.$) Cronq., 0, M(T), Q B$ 09, QB 19.
Conyza bonariensis (L.) Cronq., O, M(T), QB 19, QB 29.

Conyza albida Sprengel, $\mathbf{0}, \boldsymbol{M ( T ) , Q C} 00, Q B$ 09, PB 59.

Filago pyramidata L., F, M, QB 19, QC 00, PC 80, QB 29, PB 99.

Filago lutescens Jordan, R, M, PB 89.

Filago lutescens Jordan subsp. atlantica Wagenitz, R, M, QB 19.

Logfia gallica (L.) Cosson \& Germ., F, T(M), QB 29, PC 81, QC 10, QB 19, QC 00, PB 99.

Logfia arvensis (L.) J. Holub, R, M, QB 29.

Evax pygmaea (L.) Brot., 0, T, QB 17.

Micropus supinus L., R, M, QB 29.

Gnaphalium luteum-album L., R, I, QC 10.

Helichrysum stoechas (L.) Moench, F, T(M), QC 10, QB 09, PB 99, QB 29, PB 58.

Phagnalon saxatile (L.) Cass., F,M(T), QB 19, QB 09, PB 99, QC 00, PB 87, PB 58.

Dittrichia viscosa (L.) W. Greuter, F, $T(M)$, PB 99, QB 19, QB 29, OC 00.

Pulicaria odora (L.) Reichenb., F, $T(M), Q B$ 09, PB 99, QB 28, PC 81, QB 29.

Pulicaria dysenterica (L.) Bernh., 0, M, QB 09, QC 00.

Pulicaria paludosa Link, F, M(T), $Q B$ 19, $P B$ 88, QB 39, PB 89, PB 59, PB 99, QB 29, PC 90 .

Pallenis spinosa (L.) Cass., 0, $T(M), Q B$ 09, PB 99, PC 80, QB 29.

Asteriscus aquaticus (L.) Less., R, M, QB 29. Bidens aurea (Aiton) Sherff, R, M, QC 00.

Xanthiu spinosu $L ., 0, T(M), Q B$ 09, $P C 50$, QB 29, QB 19.

Anthemis arvensis L., F, T(M), QB 29, QB 09, QB 19, OC 10, PB 99 .

Anthemis cotula L., F, M(T), QB 09, QB 19, QB 29, QC 00.

Achillea ageratum L., F, T(M), $P B$ 99, $P C 90$, QB 19, QC 10, QB 29 .

Chamaemelum nobile (L.) All., R, T(M), PB 99, PB 58.

Chamaemelum mixtum (L.) All., F, $\mathrm{I}(\mathrm{M}), \mathrm{PC} 81$, QC 00, QB 29, PB 99, QB 19, QB 09, PB 88.

Chamaenelum fuscatum (Brot.) Vasc., 0, T(M), QB 29, QB 18, QB 28, QB 39.

Anacyclus radiatus Loisel., R, M, QB 19.

Chrysanthemun segetun L., F, M, QC 00, QB 09, PB 99.

Tanacetun parthenium (L.) Schultz Bip., R, M, QB 09 .

Daveana anthemoides Mariz, $\mathrm{Vr}, \mathrm{T}, \mathrm{QB} 29$.

Coleostephus myconis (L.) Reichenb., A, M(T), QB 09, QC 00, QB 29, QB 19, PB 99.

Leucanthenun vulgare Lam., 0, M, PB 99, QC 10, QC $00, Q B 09$.

Gymostyles stolonifera (Brot.) Tutin, R, M, QB 09. 
Doronicum plantagineum L., 0, M, QB 09, QB 29. Senecio sylvaticus $1 ., \mathrm{R}, \mathrm{I}, \mathrm{PC} 81$.

Senecio lividus L., F, M, $Q B$ 19, $Q B$ 09, $Q C$ 00, QC 10, PB 58 .

Senecio jacobaea L., $F, I(M), Q B$ 09, PB 99, QB 19.

Senecio minutus (Cav.) DC., R, M, QB 19.

Senecio vulgaris $L$., $A, T(M), Q C 10, Q B 28$, $P B$ 89, QB 38, QB 09, QB 18.

Calendula officinalis $L ., R, A, Q B 09$.

Calendula arvensis $L$., $A, T(M), Q C 00, Q B 09$, PB 99.

Carlina racemosa $L ., 0,(T), Q B 39, Q B$ 09, PB 99.

Carlina corymbosa L., F, T(M), QB 29, QB 19, PB 99, QB 39, PC 90.

Atractylis gumifera L., $0, I(M), Q B 29, P C$ 70, PB 99.

Atractylis cancellata L., R, T, PB 99, QB 39. Arctium minus Bernh., R, M, QB 19, QB 09.

Carduus tenuiflorus Curtis, $F, T(M), Q B$ 09, QB 29, QB 19, QC 00, QC 10, PC 81.

Cirsiun vulgare (Savi) Ten., R, M, QC 00.

Picnomon acarna (L.) Cass., 0, $T(M), Q B 29$, QB 09, PC 90 .

Galactites tomentosa Moench, $F, T(M), Q C 10$, PB 99, QB 09, PC 81.

Cynara algarbiensis Cosson ex Mariz, 0, $T(M)$, PB 54, PB 99.

Cynara humilis L., F, M(I), QB 29, PB 99, PB 89.

Sylibun marianu凶 (L.) Gaertner, 0, M(I), QB 19, QB 09, QC 00, QB 29.

Leuzea conifera (L.) $D C ., 0, M, Q B$ 09, $Q B$ 19, QB 29.

Mantisalca salmantica (L.) Briq. E Cavillier, A, $M(T), P C 90, Q B 29, P B 99, Q B 19$, QB 09.

Centaurea ornata Willd., F, M(I), QB 09, PB 99, QB 19, QB 29, QB 39 .

Centaurea melitensis L., F, M(I), QB 19, QB 29.

Centaurea alba L. subsp. tartesiana Talavera, $A, M(T), P B$ 99, QB 19, QC 00, QC 10.

Centaurea pullata L., R, T(M), QB 39, QB 19.

Centaurea calcitrapa L., F, I(M), QB 29, PC 90, $P B$ 99, QB 19, QB 09.

Crupina vulgaris Cass., $\mathbf{0}, \mathrm{T}(\boldsymbol{M}), Q B 29, Q B$ 09, QB 19.

Carthanus lanatus L., A, T(M), QB 19, QB 09, QB 29.

Scolymus hispanicus L., $F, T(\boldsymbol{M}), P C 90, P B$ 19, PC 50, QB 29, QB 09.

Scolymus maculatus $L ., \mathrm{Vr}, \mathrm{T}(\boldsymbol{N}), \mathrm{PC} 50$.

Cichorium intybus $L ., 0, M(I), Q B 19$.

Cichorium endivia L., $0, M(T), P B 99, Q B$ 09, QB 29, QB 19, QB 39 .

Tolpis barbata (L.) Gaertner, F, M(T), QB 29, QC 10, PC 90, PC 81.

Tolpis unbellata Bertol, R, T(H), PB 99.
Hedypnois cretica ( $L$.$) Dum-Courset, A, M(T),$ QB 19, QB 29, QC 00, QB 39, PB 99.

Hypochoeris glabra L., F, $N(T), Q C 00, Q B$ 09, QB 38, QB 39, PB 99, PB 58.

Hypochoeris radicata L., A, $M(T), Q C 00, P B$ 89, QB 09, PC 81, QB 29.

Rhagadiolus stellatus (L.) Gaertner, F, M(T), QB 09, PC 90, QC 00, PB 89, PC 70.

Urospermum picroides (L.) Schmidt, F, M(T), QC 00, QB 09, QC 10, QB 29.

Leontodon tuberosus L., $A, M(T), Q C 00, Q B$ 18, QB 28, QB 19, QB 09, QB 39, PC 90.

Leontodon taraxacoides (Vill.) Mérat subsp. longirostris Finch \& P.D. Sell, F,M(T), QB 09, QC 00, QC 10, PB 59, PC 81.

Leontodon salzmannii (Schultz Bip.) Ball, 0, $T(\boldsymbol{M})$, QB 29, QB 38, QB 19.

Picris echioides L., R, $M(\mathrm{I}), P C 90$, QC 00 , QB 09 .

Picris comosa (Boiss.) Jackson subsp. lusitanica (Schlecht.) Talavera, $0, \mathbf{M ( T ) , P B}$ 99, PC 80, PB 90, QB 29.

Scorzonera graminifolia L., $F, M(T), Q B 19$, PB 99, PC 80, QB 29.

Scorzonera crispatula (Boiss.) Boiss., R, I, PB 99.

Scorzonera laciniata L., R, M, QB 29.

Tragopogon porrifolius L., R, M, PC 90, QC 00.

Reichardia intermedia (Schultz Bip.)Coutinho, $F, M(T), P B$ 99, QB 19, QB 09, QC 00, QB 28.

Aetheorrhiza bulbosa (L.) Cass., F, H, QB 09, QB 29.

Sonchus oleraceus L., A, M(T), PB 59, QB 18 , QB 19, QB 38, QB 09, QB 39.

Sonchus asper (L.) Hill, 0, T(M), PC 60, PB 99, QB 29, QC 00, PB 59.

Sonchus tenerrimus L., 0, M, QB 09, QB 19, PB 99.

Lactuca serriola L., F, M, QB 19, QB 29, PC 80 .

Lactuca tenerrina Pourret, R, M, QB 09.

Lactuca viminea (L.) J. \& C. Presl., $0, M(T)$, QB 19, PB 88, PC 90, QB 09.

Lactuca virosa L., F, T(M), QB 09, PB 99, QB 29, QB 19, PB 59, PC 90.

Taraxacum officinale Weber, 0, M, QB 09.

Condrilla juncea L., F, T(M), QB 29, QB 09, PC 60 .

Lapsana communis L., $0, M, P B$ 99, QB 99, QB 09, QC 00, PB 89.

Crepis capillaris (L.) Wallr., F,M(T), QB 19, $P B$ 99, QC 10, QB 09, PB 59, QB 29, PC 70.

Crepis vesicaria L., $F, M(T), . Q B$ 09, QB 19, QC 00, PB 99.

Crepis foetida L., F, M, QB 19, QB 29, QB 09, PC 90.

Andryala integrifolia L., $F, T(H), Q B 29, Q B$ 09, QC 00, PB 99, PB 87. 
Andryala laxiflora DC., 0, T(M), QB 29.

\section{MONOCOIILEDONEAS}

\section{ALISMATACEAE}

Baldellia ranunculoides (L.) Parl., 0, T, PB 87.

\section{POTAMOGE TONACEAE}

Potamogeton oblongus Viv., R, I, QB 39.

Potamogeton nodosus Poiret, $R, T(M), P C 50$.

\section{LIL IACEAE}

Allium anpeloprasum L., $F, M(T), Q B$ 09, QB 29, QC 10, PC 90, QC 00, PB 99.

Allium sphaerocephalum L., R, M, QB 09.

Allium guttatum Steven subsp. sardoum (Moris) Stearn, $\mathbf{V r}, T(\boldsymbol{H}), Q B 39$.

Allium paniculatum L., R, I(H), PB 99, PC 90.

Allium pallens L., $0, T(\boldsymbol{H}), P B 59, Q B 19, Q C$ 10, QB 29.

Allium roseum L., F, M(I), QB 29, PB 89, QC 00 .

Allium chanaemoly L., R, T, PB 99, PC 90.

Allium massaessylum Batt. \& Trabut, F, M(T), PB 99, QB 09, PC 90, PB 89, QC 00.

Allium neapolitanu Cyr., R, H, QB 29, QB 09. Colchium autumale $L ., R, I(M), Q B 01$,

Merendera filifolia Camb., $\mathbf{0}, \mathbf{T}(\boldsymbol{M}), \quad Q B 39$, - QB 18, QB 17, QB 08, QB 38.

Suilax aspera L., F, T(H), PB 99, QB 09, QB 19, QB 29, PC 60.

Asparagus acutifolius L., A, I(M), QB 09, QB 29, QB 19, PC 80, QC 00.

Asparagus albus L., 0, I(H), QB 09, QC 01.

Ruscus aculeatus L., F, M(T), PC 80, QB 09, PB 99, PC 90, QB 19.

Polygonatu multifloru (L.) All., R, K, QB 09.

Polygonatum odoratu (Miller) Druce, R, $\mathbf{n}$, QB 19, PB 99.

Urginea maritima (L.) Baker, 0, T(H), PB 89, PC 70, PB 99.

Gagea foliosa (Presl.) Schult., $0, \mathbf{I}(\mathbf{M}), Q B$ 29, QB 19, QB 28, QB 39, QB 09, QB 18.

Fritillaria lusitanica Wikströn, $R, T(\boldsymbol{H}), Q B$ 29, QB 09, PB 99.

Scilla autumnalis L., $\mathbf{F}, \mathbf{I}(\mathbf{M}), Q B 38, P C 60$, PC 70, QB 17, QB 39, QB 18, QB 19.

Scilla hyacinthoides $1 ., \mathrm{R}, \mathrm{A}, Q B 09$.

Hyacinthoides hispanica (Miller) Rothm., F, M, PC 90, QB 09, PB 99.

Muscari comosu (L.) Miller, F, I(M), PC 70, QB 09, QC 00, QB 19.

Dipcadi serotinum (L.) Medicus, $0, \mathbf{M}(\mathrm{I}), Q B$ 09, QB 39, QB 38.

Ornithogalu pyrenaicu» L., $0, N(T), P B 99$, QB 29.
Asphodelus albus Miller, 0, T(M), QC 00, PB 99, QB 09.

Asphodelus aestivus Brot., 0, T(M), PB 79, QB 19.

\section{AMARILIDACEAE}

Leucojum autumnale L., F, T(M), $P C 70, Q B$ 38, QB 17.

Leucojum trichophyllum Schousboe, R, A, PB 99.

Narcissus serotinus L., 0, T(M), QB 09, QB 29, QB 39.

Narcissus bulbocodium L., $\mathbf{0}$, T(M), QB 39, QB $28, P B 67, Q B 18$.

Narcissus polyanthus Loisel, 0, T(M), QB 39, QB 09.

Narcissus jonquilla L., $0, T(M), Q B 39, Q B 38$, QB 29.

Narcissus triandrus $L$. subsp. pallidulus (Graells) D.A. Webb, R, M, QC 00.

\section{DIOSCORIACEAE}

Tamus communis L., $F, \boldsymbol{N}(\mathrm{T}), Q B$ 09, QB 29, $P B$ 99, QB 19.

\section{IRIDACEAE}

Iris sisyrinchium L., F, T(M), QB 29, PC 70, QB 39.

Iris xiphium L., $F, M(I), P B$ 99, QB 19, QB 29, PC 90, QB 09.

Iris foetidissima L., R, H, QB 09.

Iris albicans Lange, R, M, QB 09.

Iris germanica L., R, M, QB 19.

Crocus serotinus Salisb. subsp. salzmannii (Gay) Mathew, 0, I(M), PC 70, PC 80, QB 09, QC 10.

Crocus clusii Gay, R, M, QB 19.

Romulea bulbocodium (L.) Sebastiani \& Mauri, F, $T(M), Q B 19, Q B 39, Q B 28$.

Romulea raniflora Ten., R, $M(T), Q B 19$.

Gladiolus illyricus Koch, F, I(M), QB 19, PB 89, QB 09, QB 29, PC 70, QC 00.

\section{JUNCACEAE}

Juncus inflexus L., $0, T(\boldsymbol{H}), Q C \quad 00, Q B 29, Q B$ 09.

Juncus effusus L., F, I(M), QC 00, PB 59, PC 81, PB 89.

Juncus bufonius L., F, T(M), PB 99, PC 70, PC 81, QB 09, QB 29.

Juncus pigmaeus L.C.M. Richard, O, T, QB 39, PB 87.

Juncus acutiflorus Hoffm., R, T, QB 39.

Juncus articulatus L., $\mathbf{0}, \mathrm{T}(\boldsymbol{M})$, PB 88, PC 60.

Juncus obtusiflorus Hoffm., F, T(M), PB 89, QC 00 .

Luzula forsteri (Sm.) DC., R, M, PB 89, QB 09, GRAMINEAE

Lolium temulentum L., R, M, QB 29. 
Lolium perenne L., F, T(H), QB 29, PB 99, QC 10, QB 19.

Lolium rigidum Gaudin, F, $T(\boldsymbol{H}), Q B$ 29, QC 10 , QB 19, QB 09, PB 99, PC 80 .

Festuca ampla Hackes, 0, M(I), PV 87, QB 09.

Festuca paniculata (L.) Schinz \& Thell., R, $M, Q B 09$.

Vulpia ciliata Dumort., $\mathbf{R}, \mathrm{T}(\boldsymbol{M})$, QB 19, QB 09.

Vulpia geniculata (L.) Link, F, M(T), QB 19, QB 29.

Vulpia bromoides (L.) S.F. Gray, R, I(H), QB 19, QB 39 .

Vulpia muralis (Kunth) Nees, R, T(M), QB 29.

Vulpia myurus (L.) C.C. Gmelin, $\mathbf{0}, \mathbf{M}(\mathrm{T}), Q B$ 09, QC 00.

Vulpia alopecurus (Schousboe) Dumort., F, M(T), QB 29, QB 09.

Micropyru tenellus (L.) Link, $F, M(I), Q C$ $00, Q B \quad 09, Q B 19$.

Poa trivialis L., R, M, QB 29.

Poa annua L., A, M(I), PC 90, QB 09, PB 99, QC 00 .

Poa bulbosa L., F, N(T), QB 18, PC 90, QB 09, QB 39.

Desmazeria rigida (L.) Tutin, 0, I(M), QB 29, QB 09, PB 99.

Dactylis glomerata L., F, $\mathrm{I}(\mathrm{M}), \mathrm{QC} 10, \mathrm{QB} 18$, QB 19, PB 99.

Cynosorus echinatus L., F, M(T), QB 29, QB 18, QB 09, PC 80, PB 99.

Cynosorus elegans Desf., 0, A, QB 19.

Lanarckia aurea (L.) Moench, A, I(M), PC 70, QB 19, QB 39, QB 29.

Psilurus incurvus (Gouan) Schinz \& Tell, 0, T, QB 29.

Mibora minima (L.) Desv., R, I, QB 39.

Briza minor L., A, M(T), QC 10, PB 99, PC 70, QB 09, QB 29, QC 00 .

Briza maxima L., A, $M(T), Q B$ 09, QB 19, $P B$ 99, QC 00.

Melica ciliata L., O, M, PB 99, QB 19.

Melia minuta L., $F, M(T), Q B$ 19, QB 09, PB 99.

Glyceria declinata Breb., R, M, PB 99.

Bromus diandrus Roth., 0, M(I), PC 70, QB 09, QC $00, P C 81, Q B 29$.

Bronus tectorum L., 0, T(M), PC 70 .

Bronus madritensis L., R, M, QB 29.

Bronus rubens L., R, T, QC 10.

Bronus hordeaceus L., R, T(M), QB 19.

Bronus scoparius L., R, T(M), QB 09.

Brachypodium sylvaticum (Hudson) Beauv., F, M, QB 19, QC 00, QB 09.

Brachypodiun phoenicoides (L.)Roemer \& Schultes, $R, M(T), Q B 09$.

Brachypodiun distachyon (L.) Beauv., 0, M(T), QB 29, QB 19.

Elymus repens (L.) Gould, R, I(M),QB 29. Aegilops geniculata Roth, O, T, QB 19.
Aegilops triuncialis $1 ., F, M(T), Q B 29, Q B$ 19, QB 09.

Aegilops neglecta Req. ex Bertol., 0, M, QB 09, QB 19.

Hordeum murinum L., A, $\mathrm{I}(\mathrm{M}), \mathrm{QB}$ 09, QB 19, QB 29, QC 00.

Taeniatheru caput-medusae ( $L$.) Nevski, R, M, QB 29.

Avena sterilis L., F, $M(T), Q C$ 10, QC 00 . QB 09, QB 19.

Avena barbata Pott. ex Link, F, M(I), QB 29, QB 09, QB 19.

Avenula sulcata Gay ex Delastre, Vr, M, QB 09. Arrhenatherum albun (Vahl) W.D. Clayton, F, $M(T), Q B$ 09, PB 99, QB 19.

Arrhenatherum elatius Beauv. ex J. \& C. Presl subsp. bulbosu (Willd.) Schübler $\varepsilon$ Martens, R, M, QB 09.

Gaudinia fragilis (L.) Beauv., F, T(M), QB 29, QC 10.

Lophochloa cristata (L.) Hyl., 0, M, QB 19, PB 99, QB 29.

Irisetum paniceum (Lam.) Pers., R, T(M), PB 99.

Aira caryophylla L., F, T(N), QC 10, QB 19.

Aira cupaniana Guss., F, M(T), QB 29, PC 90, QB 09, QC 00, QB 19.

Molineriella minuta (L.) Rouy, F, M(I), QB 29, QB 39.

Airopsis tenella (Cav.) Ascherson \& Graebner, 0 , T(M), QC 10, PB 99 .

Anthoxanthum aristatum Boiss., F, T(N), QC 10, PC 81, QB 29.

Holcus lanatus L., F, M(I), QB 29, PB 99, QB 19, QB 09, QC 10, PC 70.

Holcus mollis L., R, M, QB 09 .

Holcus setiglumis Boiss. \& Reuter, R, A, QB 09.

Agrostis castellana Boiss. E Reuter, F, M(T), QB 19, QC 10.

Agrostis tenerrima Trin., R, M, PB 99.

Agrostis capillaris L., R, M, QB 29.

Agrostis pourretii Willd., F, T(M), PC 81, QB 29, QC 10.

Agrostis delicatula Pourret ex Lapeyr., R, T (M), QC 00.

Gastridium ventricosum (Gouan)Schinz \& Thell., 0 , M(T), QB 19, QB 29, PB 99.

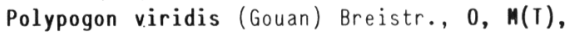
QB 29, QB 09, PB 99.

Polypogon maritimus Willd., R, M, QB 19.

Chaetopogon fasciculatus (Link) Hayek, 0, T (M), QB 18, QB 29.

Alopecurus arundinaceus Poiret, R, T, QB 29.

Phalaris coerulescens Desf., R, M, QB 29.

Phalaris aquatica L., O, T(M), QB 19, QB 29.

Phalaris canariensis L., $0, M(T), Q B$ 09, QB 18.

Phalaris paradoxa L., R, M(T), QB 19.

Stipa bromoides (L.) Dörfler, R, M, QB 09.

Stipa gigantea Link, R, M, QB 09. 
Stipa capensis Thunb., 0, T, QB 18.

Piptatherum miliaceum (L.) Cosson, F, T(M), QB 19, QB 09.

Cynodon dactylon (L.) Pers., F, T(M), QB 19, QB 29, QB 09.

Echinochloa crus-galli (L.) Beauv. F, T(M), QB 09, QB 29, PB 99, PB 88.

Digitaria sanguinalis (L.) Scop., F, $M(T)$, QB 09, PB 88, QC 00, PC 70, PC 90.

Paspalum dilatatum Poiret, $F, M(T), Q C 00$, QB 09 .

Setaria pumila (Poiret) Schultes, $0, M(T)$, QC 00, PC 60, QB 19.

Setaria viridis (L.) Beauv., 0, H, QC 00, QB 19.

Sorgum halepense (L.) Pers., 0, M, QC 00.

Hyparrhenia hirta (L.) Stapf, F, T(M), QB 39, PB 99, QB 19, QB 09.

\section{PALMAE}

Chamaerops humilis L., 0, I, QB 17.

\section{ARACEAE}

Arisarum vulgare Targ.-Tozz., A, T(M), QB 29, QB 19, QB 39, QB 09 .

Arum italicum Miller, 0, $\mathbf{\text { (T) }}(\mathrm{T}, \mathrm{PC}$ 80, PB 99, QB 19 .

Biarum arundanum Boiss. E Reuter, $R, T(\boldsymbol{M})$, PB 87, QB 29.

\section{LEMNACEAE}

Lemna minor L., 0, T(M), QB 09, PB 89, QB 19 , PC 90 .

Lemna gibba L., 0, T(M), PC 90, QB 09, QC 00.

\section{CYPERACEAE}

Cyperus longus L., 0 , $\mathrm{M}(\mathrm{I}), Q B$ 09, $P B$ 99, $Q B$ 29, QC 00 .

Cyperus fuscus. L., 0, I, QB 39, QB 29.

Cyperus flavescenș L., R, T, QB 19.

Scirpus holoschoenus L., $F, T(M), Q B$ 09, PC 90, QB 29, PB 99.

Scirpus cernuus Vahl, R, M, QB 09.

Scirpus setaceus L., R, H, PB 99.

Eleocharis palustris (L.) Roemer \& Schultes, $R, T, Q C 00$.

Carex pendula Hudson, 0, M(T), QC 00, PC 90, PB 99.

Carex remota L., R, M, QB 09.

Carex flacca Schreber, O, M, QB 19, QB 09.

Carex divisa Hudson, O, M, QB 29, QB 39.
Carex divulsa Stokes, F, M, PC 90, QC 00, QB 19, PB 89, QB 09.

Carex fusca All., 0, M(T), PC 90, QB 39.

Carex distachya Desf., R, M, QB 09.

Carex paniculata L. subsp. lusitanica (Schrank) Maire, 0, M, PB 89, QB 09.

Carex acuta L., 0, M, QB 09, QB 19.

\section{ORCHIDACEAE}

Aceras anthropophorum (L.) Ait. fil., Vr, M, QB 09 .

Spiranthes aestivalis (Poiret) L.C.M.Richard, $R, M(T), P B 89, P B 88, P C 90$.

Orchis coriophora L., R, T, QB 29.

Orchis lactea Poiret, $R, M(T), P C 90, Q B 09$.

Orchis mascula L., F, $M(T), P B 89, P C 90, Q B$ 09.

Orchis mascula L. subsp. hispanica Soó, 0 , M, QB 09.

Orchis italica Poiret, $0, T(M), Q B$ 39, QB 09, QB 29.

Orchis morio L. subsp. chanpagneuxii (Barn.) Camus, Vr, M(I), QB 19.

Dactylorhiza sambucina (L.) Soó, Vr, M, QB 09.

Dactylorhiza incarnata (L.) Soó, R, M(T), QC 00, PC 70.

Dactylorhiza sulphurea (Link) Franco, R, A, QB 19.

Cephalanthera longifolia (L.) Fritsch, F, M (I), PB 99, QB 09, QB 19, PC 90, QC 00.

Epipactis helleborine (L.) Crantz, F, M(I), QB 09, PB 99, PB 89, QB 19, PC 81, PC 90, QC 00.

Serapias parviflora Parl., R, M, QB 19, QB 09.

Serapias voneraceae (Burm.) Briq., $0, \mathbf{I}(\mathbf{M})$, QB 29.

Serapias lingua L., $F, T(M), Q B$ 09, QB 29, QB 39 , QB 19, QC 00.

Ophrys dyris Maire, R, M, QB 09.

Ophrys lutea (Gouan) Cav., R, A, QB 19.

Ophrys apifera Hudson, 0, $\mathbf{M}$ QB 29, PB 89, QB 09 , PC 70.

Ophrys tenthredinifera Willd., O, T, QB 39.

Ophrys fusca Link, R, M, QB 09.

Ophrys fusca Link subsp.omegaifera (Fleischme) E. Nelson, R, H, QB 09.

Limodorum abortivum (L.) Schwartz, F, H, QB 09, QB 29, PC 90, QB 19.

Neotinea maculata (Desf.) Stearn, F, $\boldsymbol{\text { (T) }}(\mathrm{T}), Q B$ 09, QB 19, PC 70.

\section{B I BL IOGRAF IA}

CABEZUDO, B. - 1978a- Nota corológica sobre la flora de Huelva.IV. Lagascalia, 7: 173-178.

CABEZUDO, B. -1978b- Pteridófitos de la provincia de Huelva. Lagascalia, $8: 2-12$. 
RIVAS GODAY, S. -1964- Vegetación y flórula de la cuenca extremeña del Guadiana. Madrid.

RIVAS-MARTINEZ, S. -1979- Brezales y jarales de Europa Occidental. Lazaroa, 1: 5-127.

RIVAS-MARTINEZ, S. -1982a- Mapas de las series de vegetación de Madrid. Madrid.

RIVAS-MARTINEZ, S. -1982b- Etages bioclimatiques, secteurs chrologiques et séries de végétation de l'Espagne méditerranéenne. Ecol. Mediterranea, $8(1 / 2): 275-288$.

RIVERA, J. \& CABEZUDO, B. -1981- Notas taxonómicas y corológicas sobre la flora de Andalucía Occidental. Notas breves. Lagascalia, 10: 95127.

SALVO, A.E. \& CABEZUDO, B. -1984- Lista comentada de los pteridófitos de Andalucía. Acta Bot. Malacitana, 9: 133-146.

TUTIN, T.G. \& cols. (eds.) -1964-80- Flora Europaea. Cambridge. 